\title{
Localized in-gap states and quantum spin Hall effect in Si-doped InAs/GaSb quantum wells
}

\author{
Dong-Hui Xu, ${ }^{1}$ Jin-Hua Gao, ${ }^{2}$ Chao-Xing Liu, ${ }^{3}$ Jin-Hua Sun, ${ }^{1}$ Fu-Chun Zhang, $,{ }^{1,4},{ }^{*}$ and Yi Zhou ${ }^{1, \dagger}$ \\ ${ }^{1}$ Department of Physics, Zhejiang University, Hangzhou 310027, China \\ ${ }^{2}$ Department of Physics, Huazhong University of Science and Technology, Wuhan, Hubei, China \\ ${ }^{3}$ Department of Physics, The Pennsylvania State University, University Park, Pennsylvania 16802-6300, USA \\ ${ }^{4}$ Department of Physics, and Center of Theoretical and Computational Physics, The University of Hong Kong, Hong Kong, China
}

(Received 2 November 2013; revised manuscript received 21 April 2014; published 7 May 2014)

\begin{abstract}
We study localized in-gap states and quantum spin Hall effect in Si-doped InAs/GaSb quantum wells. We propose a model with donor and/or acceptor impurities to describe Si dopants. This model shows in-gap bound states and wide conductance plateau with the quantized value $2 e^{2} / h$ in light dopant concentration, consistent with recent experiments by Du et al. (Lingjie Du et al., arXiv:1306.1925). We predict a conductance dip structure due to backward scattering in the region where the localization length $\xi$ is comparable with the sample width $L_{y}$ but much smaller than the sample length $L_{x}$.
\end{abstract}

DOI: 10.1103/PhysRevB.89.195104

PACS number(s): 72.15.Rn, 72.20.-i, 73.20.Fz, 73.63.Hs

\section{INTRODUCTION}

The quantum spin Hall (QSH) insulator is the first theoretically predicted two-dimensional (2D) time reversal invariant topological insulator (TI) that manifests topologically nontrivial edge states. It is characterized by topologically robust gapless (spin polarized) counter-propagating states at the edge while there exists an energy gap in the bulk [1,2]. The first realistic material for QSH insulators was proposed by Bernevig et al. [3] in a semiconductor $\mathrm{CdTe} / \mathrm{HgTe} / \mathrm{CdTe}$ quantum well (QW), which was later confirmed in transport experiments [4]. Soon after the discovery, there have been world-wide activities in 3D TIs [5-7]. Although nonlocal measurements have exhibited edge transports [8], large conductance fluctuation in the QSH regime of HgTe QWs has never been well understood.

The second example of QSH insulators is the type II InAs/GaSb QW proposed by Liu et al. [9], which has been realized in experiments [10-12]. A unique feature of this system is that the conduction band bottom of bulk InAs is about $150 \mathrm{meV}$ lower than the valence band top of bulk GaSb. Therefore, when InAs and GaSb together form a quantum well structure, the electron subband from the InAs layer can be lower than the heavy hole subband from the GaSb layer [see Fig. 1(a)], forming a so-called "inverted band structure." The position of electron and hole subbands in this system can be controlled by varying the thickness of InAs and GaSb layers, and the band inversion occurs in a large range of the thickness of the QW. The hybridization of electron and hole states in the QW opens a minigap of $40 \sim 60 \mathrm{~K}$. When the Fermi energy lies in the minigap, charge transport is dominated by topological edge modes, which can be extracted from conductance measurements $[10,11]$. However, early experiments exhibit conductance larger than the expected values of $\frac{2 e^{2}}{h}$ for a QSH insulator, presumably due to residual bulk currents carried by disorder induced (extended) states inside the minigap.

Very recently, Du et al. [13] introduced light Si dopants $\left(\sim 10^{11} \mathrm{~cm}^{-2}\right)$ in InAs/GaSb QWs. Si atoms serve as donors in

\footnotetext{
*fuchun@hku.hk

†yizhou@zju.edu.cn
}

InAs and acceptors in GaSb. They have found that Si doping suppresses the bulk conductance and induces a mobility gap of $26 \mathrm{~K}$ inside the minigap. They have observed a wide plateau of quantized longitudinal conductance of $G_{0}=2 e^{2} / h$ within $1 \%$ as the front gate voltage changes. Therefore, it is natural to ask how the Si doping induces the quantized conductance plateau.

Theoretically, the disorder effect has been numerically investigated in the context of graphene [14] and HgTe QWs $[15,16]$. It was found that under magnetic fields, disorder can induce localization behavior of helical edge states, which is the origin of the cusplike feature in magnetotransport of HgTe QWs [16]. In addition, strong disorder may drive an ordinary insulating state to a topological Anderson insulator (TAI) $[15,17]$, which may be understood as a band inversion caused by effective mass renormalization [18]. (Energy band renormalization is distinct in $\mathrm{HgTe}$ and $\mathrm{InAs} / \mathrm{GaSb}$, see Appendix A for details.) The phenomenon of TAI requires very strong impurity scattering potential or high impurity concentration to localize bulk states and to renormalize the effective mass.

In this paper, we study the effect of Si dopants in InAs/GaSb QW in the band inverted region. A single Si dopant serves as a donor or acceptor and introduces a bound state in the minigap, similar to a hydrogenic bound state in conventional semiconductors. Note that weak disorder leads to localization in $2 \mathrm{D}$ in the thermodynamic limit. For a mesoscopic sample of length $L_{x}$, the nature of localization depends on the localization length $\xi$. When $\xi<L_{x}$ in QWs, a bulk mobility gap is opened and only the edge state transport remains. We use Landauer-Büttiker formalism to calculate conductance and show a wide plateau in quantized conductance of $2 e^{2} / h$. Our theory explains the observed mobility gap and quantized conductance in the experiment of Du et al.

\section{MODEL HAMILTONIAN}

The bulk of InAs/GaSb QW can be well described by a four band tight-binding model on a square lattice where four relevant atomic states $\{|E+\rangle,|E-\rangle,|H+\rangle,|H-\rangle\}$ are involved. $E$ and $H$ mark electron and hole states, respectively, and \pm correspond to pseudospins. This tight-binding Hamiltonian 


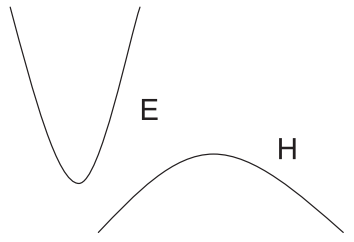

(a)

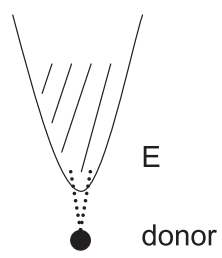

(c)

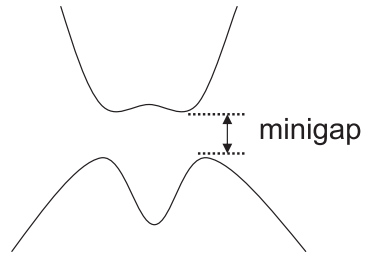

(b)

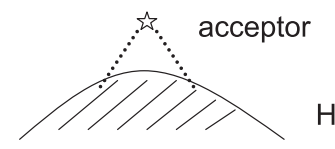

(d)
FIG. 1. (a), (b) Band inversion in InAs/GaSb. (a) Conduction band of InAs (denoted by $E$ ) is lower than the valence band of GaSb (denoted by $H$ ). (b) The hybridization of the electron and hole states opens a minigap. (c), (d) Schematic impurity level and impurity scattering. (c) Si atoms serve as donors in InAs. (d) Si atoms serve as acceptors in GaSb.

can be derived from a corresponding $k \cdot p$ Hamiltonian [9] and reads

$$
H_{0}=\sum_{i \sigma \alpha} V_{\alpha \sigma} c_{i \alpha \sigma}^{\dagger} c_{i \alpha \sigma}+\sum_{i \tau \sigma \alpha \beta} t_{\alpha \beta}^{\tau \sigma} c_{i \alpha \sigma}^{\dagger} c_{i+\tau \beta \sigma},
$$

where $i$ is the site labeling, $\tau= \pm \hat{x}, \pm \hat{y}$ denotes the four nearest neighbors bond, $\sigma= \pm$ is for pseudospin, and $\alpha, \beta=$ $E, H$ is the orbital index. The pseudospin is a good quantum number. In the sub-Hilbert space spanned by $\{|E \sigma\rangle,|H \sigma\rangle\}$, $V_{\alpha \sigma}$ is a diagonal matrix

$$
V_{\sigma}=\left(\begin{array}{cc}
C-4 D+M-4 B & 0 \\
0 & C-4 D-M+4 B
\end{array}\right)
$$

and $t_{\alpha \beta}^{\tau \sigma}$ is given by the following matrix form:

$$
\begin{aligned}
t^{ \pm \hat{x} \sigma} & =\left(\begin{array}{cc}
D+B & \mp i \sigma A / 2 \\
\mp i \sigma A / 2 & D-B
\end{array}\right), \\
t^{ \pm \hat{y} \sigma} & =\left(\begin{array}{cc}
D+B & \pm A / 2 \\
\mp A / 2 & D-B
\end{array}\right),
\end{aligned}
$$

where $A, B, C, D$, and $M$ are parameters determining the band structure and will be given later. The lattice constant in InAs/GaSb is about $6 \AA$. However, as an effective tightbinding model derived from the $k \cdot p$ Hamiltonian, we can use a different lattice constant by properly choosing parameters. In this paper, we shall set the lattice constant as $a=20 \AA$ and choose $A=0.0185 \mathrm{eV}, B=-0.165 \mathrm{eV}, C=0, D=$ $-0.0145 \mathrm{eV}$, and $M=-0.0078 \mathrm{eV}$. This set of parameters corresponds to the set of $k \cdot p$ parameters used in Ref. [9]. For simplicity, we neglect the terms describing bulk and structure inversion asymmetry [9], which are inessential for the physics of QSH effect.
The k component of Hamiltonian (1) can be expressed as

$$
H_{0}(\mathrm{k})=\epsilon(\mathrm{k}) \mathbf{I}+\left(\begin{array}{cc}
h_{0}(\mathrm{k}) & 0 \\
0 & h_{0}^{*}(-\mathrm{k})
\end{array}\right),
$$

where $h_{0}(\mathrm{k})$ is a $2 \times 2$ matrix,

$$
h_{0}(\mathrm{k})=\left(\begin{array}{cc}
M(\mathrm{k}) & A\left(\sin k_{x}-i \sin k_{y}\right) \\
A\left(\sin k_{x}+i \sin k_{y}\right) & -M(\mathrm{k})
\end{array}\right),
$$

I is the $4 \times 4$ identity matrix, $M(\mathrm{k})=M-2 B\left(2-\cos k_{x}-\right.$ $\left.\cos k_{y}\right)$ and $\epsilon(\mathrm{k})=C-2 D\left(2-\cos k_{x}-\cos k_{y}\right)$. Equation (4) recovers the $k \cdot p$ Hamiltonian at small $k$ [9]. According to $\mathrm{Fu}$ and Kane's criterion for TIs, topological nontrivial states exist when $0<M / 2 B<2$ [19].

Now we consider the disorder induced by Si dopants, which serve as donors in InAs ( $E$ band) and acceptors in $\mathrm{GaSb}(H$ band). A donor attracts $E$ band electrons and its interaction with $H$ band can be neglected. Similarly, an acceptor provides a repulsive scattering potential to $H$ band while leaving $E$ band noninteracted [20]. To model the disorder effect of Si dopants in InAs/GaSb, we introduce the following on-site impurity Hamiltonian,

$$
H_{\mathrm{imp}}=-\sum_{i \in R_{d}, \sigma} V_{i} c_{i, E \sigma}^{\dagger} c_{i, E \sigma}+\sum_{i \in R_{a}, \sigma} V_{i} c_{i, H \sigma}^{\dagger} c_{i, H \sigma},
$$

where $R_{d}$ and $R_{a}$ denote the site sets for donors and acceptors, respectively. $V_{i}$ is positive and distributes randomly within a range of $\left(V_{\min }, V_{\max }\right)$. While this is a simplified model for the charge impurity potentials, it captures the basic physics in $\mathrm{Si}$ dopant systems. As we will see below, this type of disorder has the largest efficiency in inducing in-gap bound states, which are essential for the localization physics. For the lattice constant $a=20 \AA$ used in this paper, $10^{11} \mathrm{~cm}^{-2} \mathrm{Si}$ dopant corresponds to $0.4 \%$ impurity in our model.

\section{SINGLE IMPURITY}

We begin with a single impurity problem. In a semiconductor, a dopant can always induce in-gap bound states because of the long-ranged Coulomb attraction. In our model, the impurity potential is a short-ranged and delta-function-like potential in 2D. At first glance, this kind of impurity potential does not support bound states unless it exceeds a threshold. But this is not true here because the density of states (DOS) is singular at the band edge in the clean system [see Fig. 3(b)]. In this case, even infinitesimal attractive potential will give rise to in-gap bound states [21]. So our model catches the in-gap physics correctly although it uses a simplified impurity potential. To examine this point, we diagonalize the Hamiltonian $H=$ $H_{0}+H_{\text {imp }}$ numerically on a $50 \times 40$ lattice and find that an in-gap state appears in the presence of a single impurity. For simplicity, we only show the results on donors, and the acceptor situation is similar. The energy difference $\Delta E$ between the in-gap state and band edge for a pure system is plotted in Fig. 2(a) as a function of the attractive potential $V$. It is clear that there are bound states at any attraction strength. The density distributions for the corresponding in-gap bound states are shown in Figs. 2(b)-2(d). The in-gap state becomes more and more localized as the attractive potential increases. For hole states in the valence band, an acceptor carries a negative 

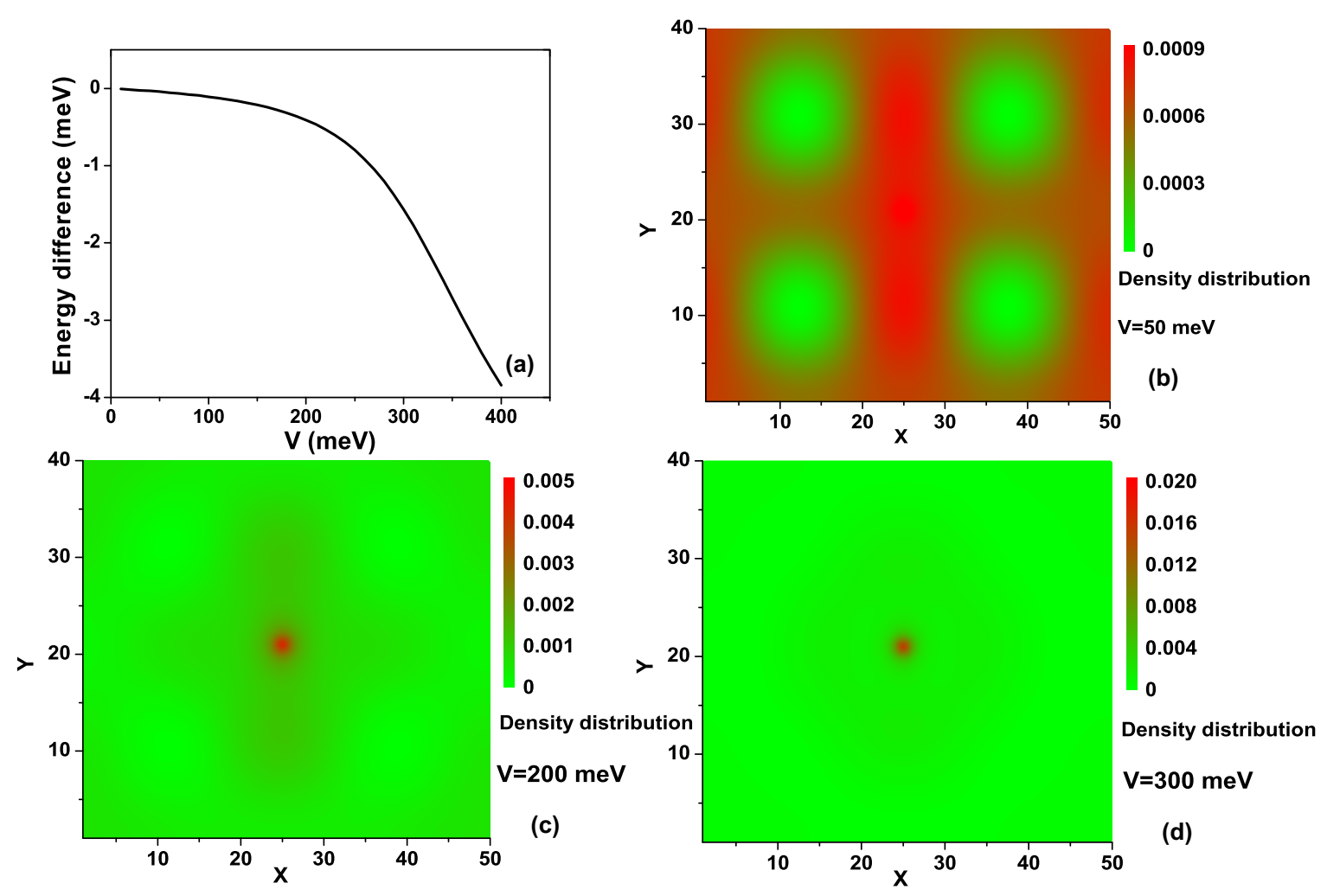

FIG. 2. (Color online) (a) Binding energy of single impurity (donor) state. (b)-(d) Local density of states of bound states induced by a single impurity. $V=50,200,300 \mathrm{meV}$ in (b)-(d).

charge and is attractive to holes while repulsive to electrons. It will also induce in-gap bound states. Therefore, our choice of signs of $V_{i}$ in $H_{\text {imp }}$ in Eq. (6) always gives rise to in-gap bound states.

\section{LOCALIZATION IN BULK}

We continue to study the localized states in bulk in the presence of many Si dopants. We consider half of the $\mathrm{Si}$ dopants are donors and the other half are acceptors. Hereafter we set $V_{\min }=200 \mathrm{meV}$ and $V_{\max }=300 \mathrm{meV}$ unless specified otherwise. To investigate the localization problem, we calculate DOS and conductance $G=G_{x x}$ by using the recursive Green's function method [22] and Landauer-Büttiker-FisherLee formula [23]. We consider a strip geometry consisting of a $L_{x} \times L_{y}$ rectangular disorder region and two semi-infinite doped metallic leads connected to the rectangle along the $x$ direction. This setup allows us to study the transport coefficients with both open boundary condition (OBC) and periodic boundary condition (PBC) along the $y$ direction. We shall utilize PBC to study bulk states and OBC to study edge states.

The results are shown in Fig. 3. There is a window in the Fermi energy, $E_{F} \sim 0.2-2.4 \mathrm{meV}$, where DOS is finite but the conductance vanishes. This means that there are fully localized in-gap states with $\xi \ll L_{x}$ in this regime. For the regime $-3.0 \mathrm{meV}<E_{F}<0.2 \mathrm{meV}$ or $2.4 \mathrm{meV}<E_{F}<$ $4.0 \mathrm{meV}$, although the Fermi energy is still in the bulk gap, the conductance is nonzero. This is due to the finite size effect. Namely, when the localization length $\xi$ is comparable with or larger than the system size $L_{x}$, the size effect become considerable and bulk transport is allowed. In Fig. 3(d), we increase $L_{x}$ and the regime for zero conductance is also enlarged.

The localized in-gap states do not contribute to the conductance but to the DOS revealed in capacity measurements. The existence of a large amount of localized in-gap states in DOS in our simulation qualitatively agrees with the capacity measurement in experiments [13].

We remark that our sharp impurity potential scenario for $\mathrm{Si}$ dopant is very different from the weak localization scenario. Firstly, increasing impurity concentration will result in a finite bulk conductance due to the formation of impurity bands. Secondly, smooth disordered potential may give rise to in-gap "extended" states with a long localization length $\xi \gg L_{x}$ due to large DOS at the band edge. Correspondingly, the mobility gap becomes smaller than the minigap in the present system. These extended states may be further localized by $\mathrm{Si}$ dopants described by a sharp impurity potential. (See Appendix C for details.) This may explain the experiment by Du et al., where the bulk conductance is finite in the absence of the Si doping and vanishes as $\mathrm{Si}$ impurities are introduced.

\section{EDGE TRANSPORT}

When the bulk states are localized by impurities, i.e., $\xi \ll L_{x}$, the transport is entirely dominated by the edge channels, as clearly seen in Fig. 4(a). In the regime $0.2 \mathrm{meV}<$ $E_{F}<2.4 \mathrm{meV}$, a wide conductance plateau with the quantized value $\frac{2 e^{2}}{h}$ emerges for $\mathrm{OBC}$ while bulk conductance is zero and all bulk states are localized for PBC. In this regime, the 

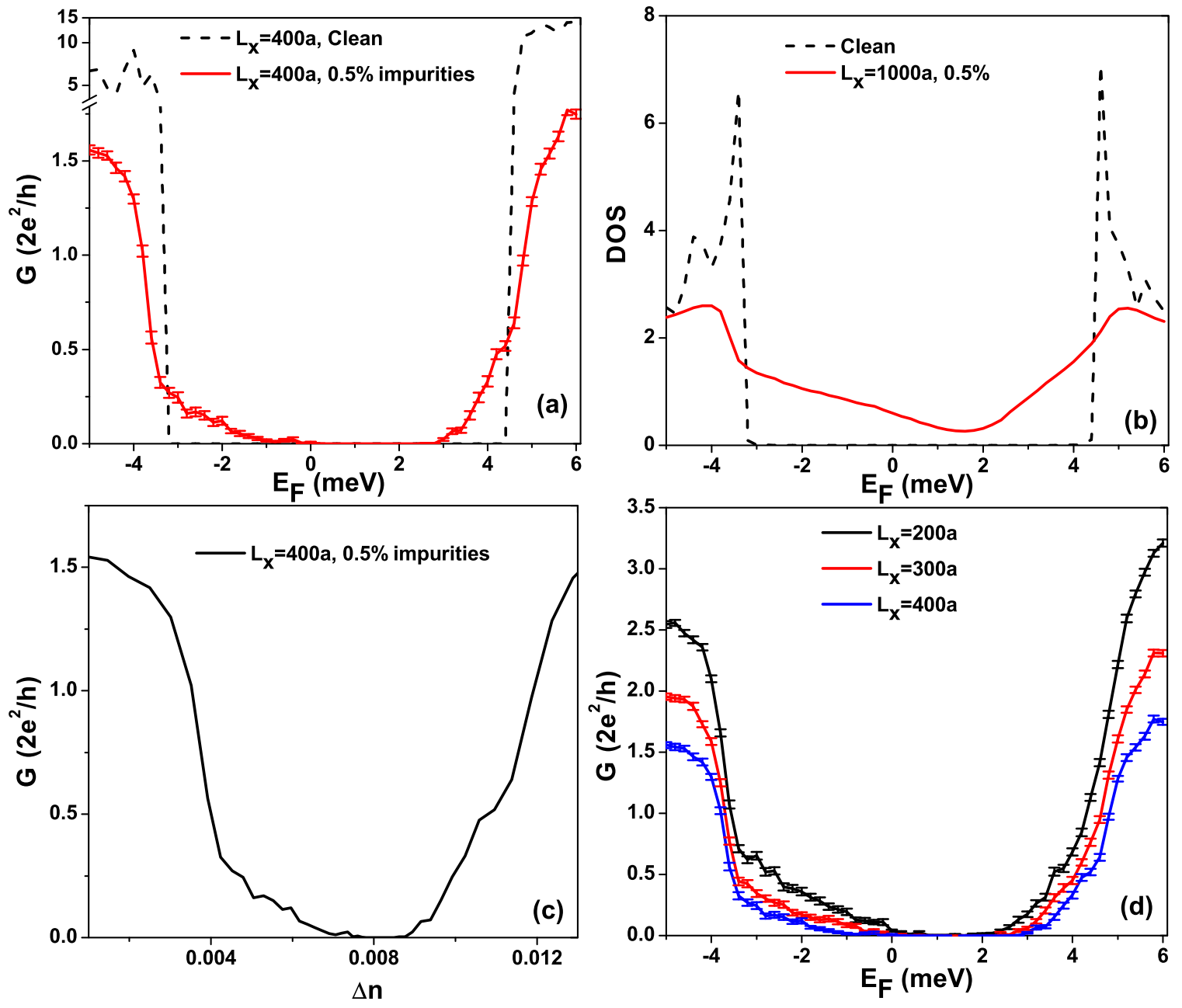

FIG. 3. (Color online) Conductance and DOS in the presence of impurities. The strip width is set to $L_{y}=200 a$. The concentration of impurity is $0.5 \%$. (a) Conductance for clean and impure samples with $L_{x}=400 a$. (b) Lead-free DOS for clean and impure samples with $L_{x}=1000 a$. (c) Conductance as the function of electron density with $L_{x}=400 a$. (d) Conductance of different samples with $L_{x}=400 a$ (blue), $300 a$ (red), and 200a (black).

localization length, $\xi \equiv-2 \lim _{L_{x} \rightarrow \infty} L_{x}\left\langle\ln G / G_{0}\right\rangle^{-1}$ [24], is found to be much smaller than both the length $L_{x}$ and width $L_{y}$ of the system, as shown in Fig. 4(b).

When the Fermi energy is tuned to the regime $-3.0 \mathrm{meV}<$ $E_{F}<0.2 \mathrm{meV}$ or $2.4 \mathrm{meV}<E_{F}<4.0 \mathrm{meV}$, we find that although the bulk conductance increases according to the calculation with $\mathrm{PBC}$, the total conductance for a system computed with $\mathrm{OBC}$ decreases at the plateau edge. This conductance dip is a finite size effect. In this regime, the localization length $\xi$ increases rapidly and when $L_{y} \sim \xi \ll$ $L_{x}$, the electron at one edge state can interfere with in-gap bound states in the bulk and will be scattered to the opposite edge, resulting in a significant reduction of edge currents. We emphasize here that the penetration length of the edge states in a clean system is quite small for the present parameters, which can not induce direct hybridization between two edge states. The backscattering is mediated by in-gap bound states.

For a given impurity concentration, the dip structure in conductance may smooth out by increasing sample width $L_{y}$ or decreasing sample length $L_{x}$, because the former will reduce the coupling between edge states and in-gap bulk states, while the later will increase the bulk conductance. Figs. 4(c), 4(d), and 4(a) show how this dip structure emerges with increasing $L_{x}$ and fixed $L_{y} \sim \xi$. This predicted dip structure in conductance under the condition $L_{y} \sim \xi \ll L_{x}$ may be examined in future experiments.

\section{COMPARISON WITH EXPERIMENTS AND SUMMARY}

Now we shall relate our numerical simulation on the localization effect to the recent experiment of InAs/GaSb QWs with Si doping. Experimentally, the conductance quantization is only observed at low temperatures. The localization length $\xi$ is temperature dependent. At high temperature, $\xi \gg L_{x}$, the impurity state is extended, and conductance is higher and not quantized. At low temperature, $\xi \ll L_{x}$ for the states deep in the band gap. As a result, the system starts to show insulating behavior. This corresponds to the development of the mobility gap observed by both the bulk conductance measurement in a Carbino disk and the electric capacity measurement. In this regime, for a finite sample, a robust quantized conductance 

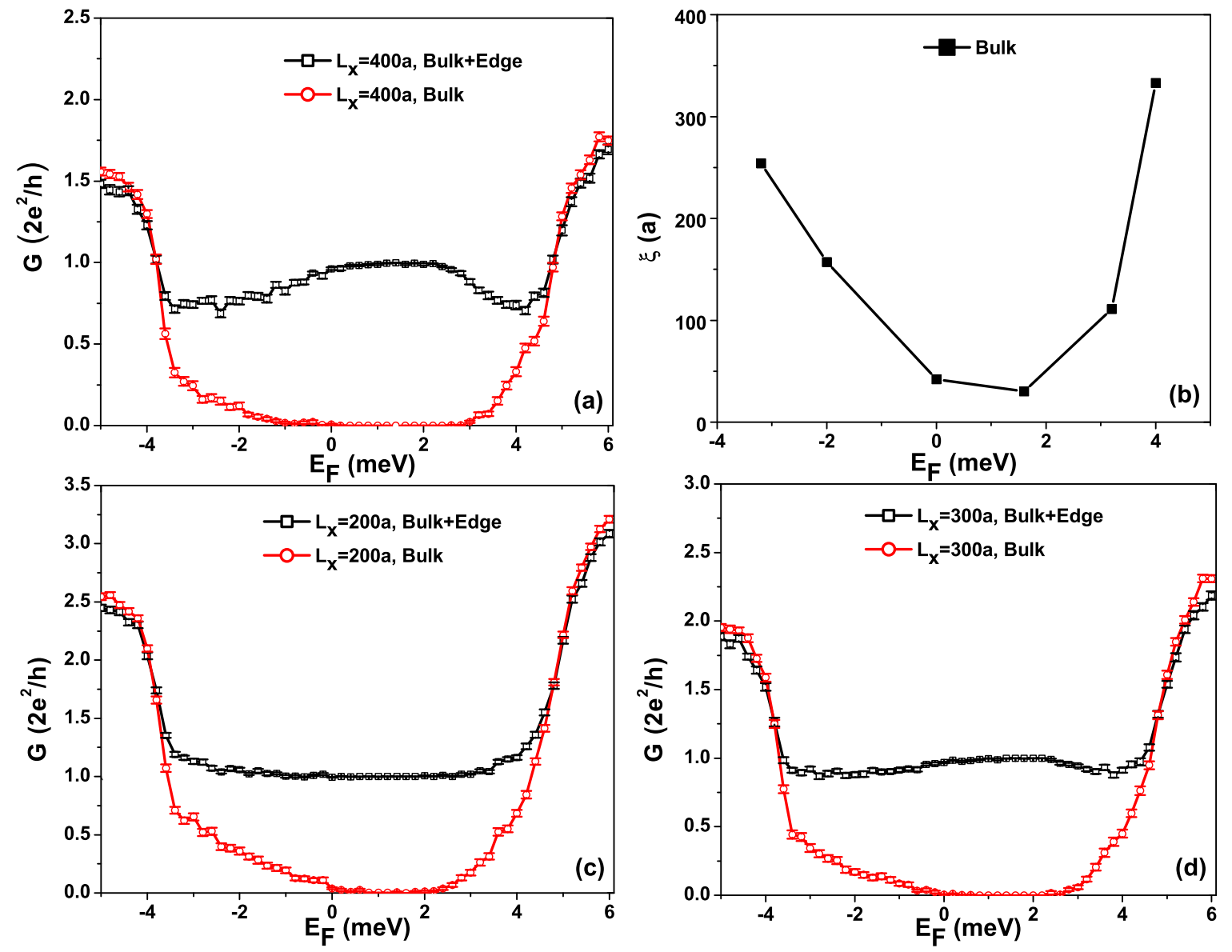

FIG. 4. (Color online) Quantized conductance plateau and localization length in a strip with width $L_{y}=200 a$ and $0.5 \%$ impurity. (a) Conductance in a $400 \times 200$ sample. (b) Localization length as a function of Fermi energy. (c) Conductance in a $200 \times 200$ sample. (d) Conductance in a $300 \times 200$ sample.

plateau appears due to the edge transport and the system enters into the QSH regime.

Finally, we would like to emphasize the uniqueness of disorder effect in InAs/GaSb QWs. In conventional materials, even smooth disorder could lead to a large mobility gap. Therefore, the precise quantization conductance plateaus can be easily observed in the quantum Hall effect [25]. In contrast, our numerical calculations have shown that the mobility gap strongly depends on the strength and types of disorders in $\mathrm{InAs} / \mathrm{GaSb} \mathrm{QWs}$. Thus, light Si doping is required to introduce in-gap bound states efficiently to form a mobility gap at a low temperature. Our theory explains the essential role of in-gap states for the highly quantized conductance plateau observed in Du et al.'s experiment at zero magnetic field, and examined conditions for the accurate quantization in the QSH systems. We note that the experiments of Du et al. also report the extreme robustness of the quantization against external magnetic field, which remains to be a theoretical challenge for our future work.

\section{ACKNOWLEDGMENTS}

We thank R. Du for many insightful discussions on their experiments, and S. Q. Shen and M. Ma for discussions in the early stage of the project. This work is partially supported by National Basic Research Program of China (No. 2011CBA00103/2014CB921201), NSFC (No. 11374256/11274269), the Fundamental Research Funds for the Central Universities in China, and HK RGC/GRF Grant No. 701010.

\section{APPENDIX A: BAND RENORMALIZATION BY ANDERSON DISORDER: SELF-CONSISTENT BORN APPROXIMATION}

To compare different band renormalization effect by Anderson disorder between $\mathrm{HgTe}$ and $\mathrm{InAs} / \mathrm{GaSb} \mathrm{QWs}$, we use self-consistent Born approximation to investigate the following random onsite potential

$$
H_{\mathrm{I}}=\sum_{i \sigma \alpha} V_{i} c_{i \alpha \sigma}^{\dagger} c_{i \alpha \sigma},
$$

where $V \in[-W / 2, W / 2]$ with disorder strength $W$. This type of impurity will renormalize the energy band through the self energy, which is defined as

$$
\left(E_{\mathrm{F}}-h_{0}-\Sigma\right)^{-1}=\left\langle\left(E_{\mathrm{F}}-h\right)^{-1}\right\rangle,
$$

where $\langle\cdots\rangle$ denotes the disorder average. Here $\Sigma$ is a $2 \times 2$ matrix which can be decomposed into Pauli matrices: $\Sigma=$ $\Sigma_{\mu} \sigma_{\mu} \mu=0,1,2,3$. The renormalized topological mass and 
chemical potential are then given by

$$
\bar{M}=M+\operatorname{Re} \Sigma_{3}, \quad \bar{E}_{\mathrm{F}}=E_{\mathrm{F}}-\operatorname{Re} \Sigma_{0},
$$

where $M$ and $E_{F}$ are bare mass and chemical potential, respectively.

In the self-consistent Born approximation, we keep the self energy up to the second order of $W$ in the spirit of perturbation. The self energy is given by

$$
\Sigma=\frac{W^{2}}{12} \sum_{\mathbf{k}}\left[E_{\mathrm{F}}+i 0^{+}-h_{0}(\mathbf{k})-\Sigma\right]^{-1} .
$$

The band gap $E_{\text {gap }}$ is determined by the upper band edge $E_{u}$ and the lower band edge $E_{l}$ through $E_{\text {gap }}=E_{u}-E_{l}$, where $E_{u}$ reads

$$
E_{u}=\min \left[\epsilon(k)+\operatorname{Re} \Sigma_{0}+\sqrt{\bar{M}^{2}(k)+A^{2} \sin ^{2} k}\right],
$$

and $E_{l}$ reads

$$
E_{l}=\max \left[\epsilon(k)+\operatorname{Re} \Sigma_{0}-\sqrt{\bar{M}^{2}(k)+A^{2} \sin ^{2} k}\right],
$$

with $\bar{M}(k)=\bar{M}-2 B\left(2-\cos k_{x}-\cos k_{y}\right)$.

To compare HgTe with InAs/GaSb, we choose the following bare band parameters for HgTe QW: $A=0.0729 \mathrm{eV}, B=$ $-0.02744 \mathrm{eV}, C=0, D=-0.02048 \mathrm{eV}$, and $M=-0.01 \mathrm{eV}$, while we use the same parameters as in the main text for InAs/GaSb QW, namely, $A=0.0185 \mathrm{eV}, B=-0.165 \mathrm{eV}$, $C=0, D=-0.0145 \mathrm{eV}$, and $M=-0.0078 \mathrm{eV}$. Figure 5 shows the renormalized band edges by disorder average based on self-consistent Born approximation. One sees that the band gap in InAs/GaSb QW will be narrowed in weak disorder strength in contrast to $\mathrm{HgTe} \mathrm{QW}$ where the band gap is enlarged by disorder. This indicates that it is easier to induce in-gap states by disorder in InAs/GaSb than that in HgTe.

\section{APPENDIX B: THE FORMATION OF IMPURITY BAND}

In the weak localization picture, the localization length will decrease as impurity concentration increases. In contrast, localized states caused by the strong localization mechanism proposed in the main text will become more and more "extended" when impurity concentration increases, because the wave function overlap between neighboring localized states will increase. Thus the localized states induced by individual impurity will form an impurity band when the impurity concentration is large enough. This impurity band will contribute to bulk transport, resulting in finite bulk conductance as shown in Fig. 6.

\section{APPENDIX C: LOCALIZATION OF IN-GAP "EXTENDED" STATES INDUCED BY SMOOTH DISORDERS}

In addition to sharp impurity potential induced by dilute $\mathrm{Si}$ dopants, one may consider smooth disorder potential commonly used in quantum Hall and other systems. We find that smooth disorders may introduce in-gap "extended" states, or to be precise, localized states with localization length $\xi$ much longer than sample length $L_{x}$. Moreover, these in-gap "extended" states will be localized by sharp impurity potential induced by dilute Si dopants.
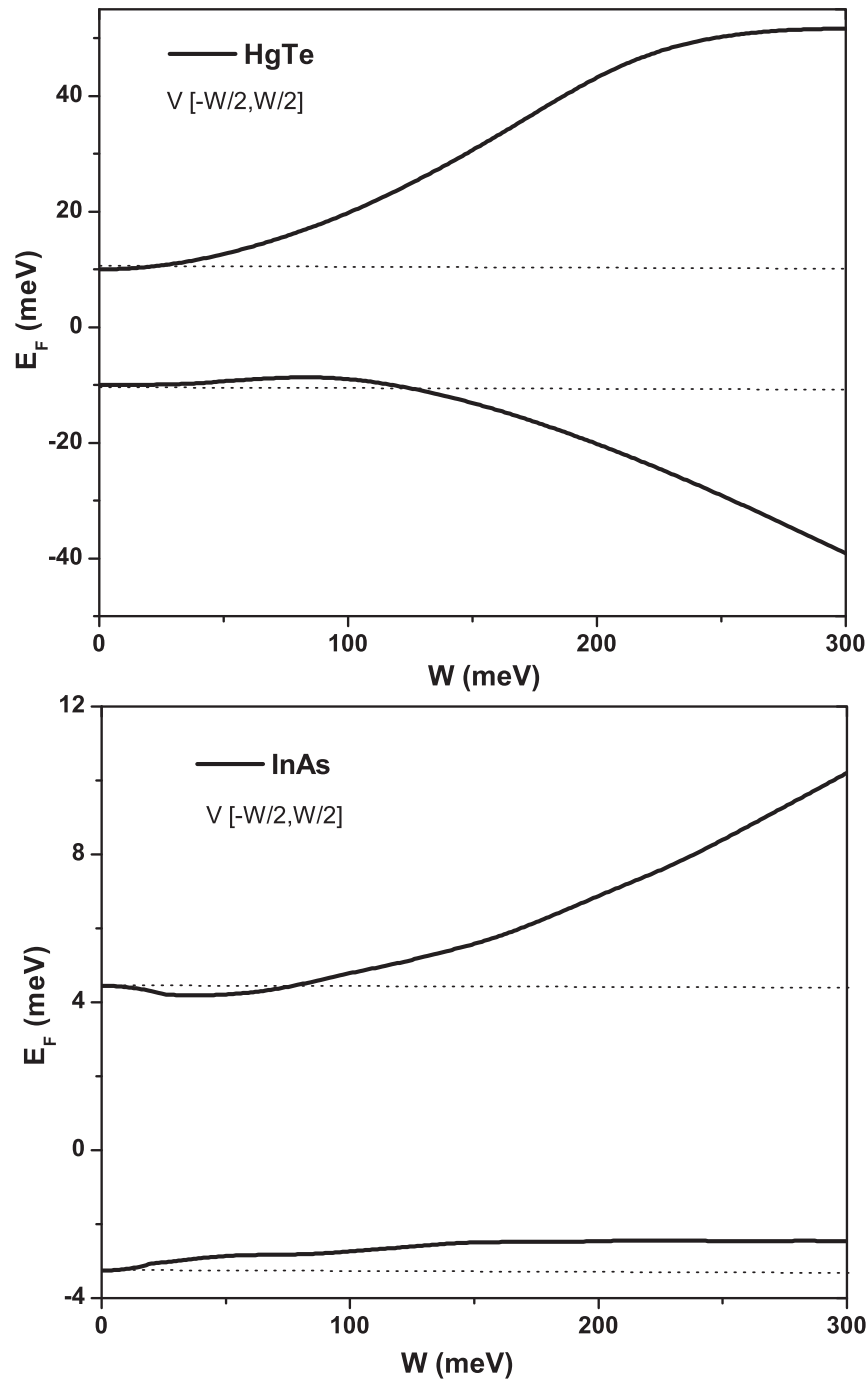

FIG. 5. Energy band renormalization by Anderson disorder. (a) Upper and lower band edges in HgTe QW. (b) Upper and lower band edges in InAs/GaSb QW.

To illustrate these effects, we use a random Gaussian potentials $H_{G}$ to model smooth disorder,

$$
H_{G}=\sum_{i, \sigma} V_{G}(i)\left(c_{i, E \sigma}^{\dagger} c_{i, E \sigma}+c_{i, H \sigma}^{\dagger} c_{i, H \sigma}\right),
$$

with

$$
V_{G}(i)=\sum_{j \in R_{G}} V_{0}(j) \exp \left[-\frac{\left(\vec{r}_{i}-\vec{r}_{j}\right)^{2}}{2 b^{2}}\right],
$$

where $R_{G}$ is the collect of site $j$ where the Gaussian peaks locate, $b$ is a constant to describe the width of Gaussian potential, and $V_{0}$ distributes uniform randomly in $\left[-V_{s}, V_{s}\right]$. We choose $R_{G}$ to contain $10 \%$ sites in the whole lattice, $b=5 a$ and $V_{s}=10 \mathrm{meV}$ to generate smooth disorder potential. The sharp impurity potential induced by $\mathrm{Si}$ is described by random delta-function-like potential $H_{\mathrm{imp}}$ as in the main text with $V_{\min }=300 \mathrm{meV}$ and $V_{\max }=400 \mathrm{meV}$. We use $4 \%$ sharp impurity concentration to illustrate the localization effect. 


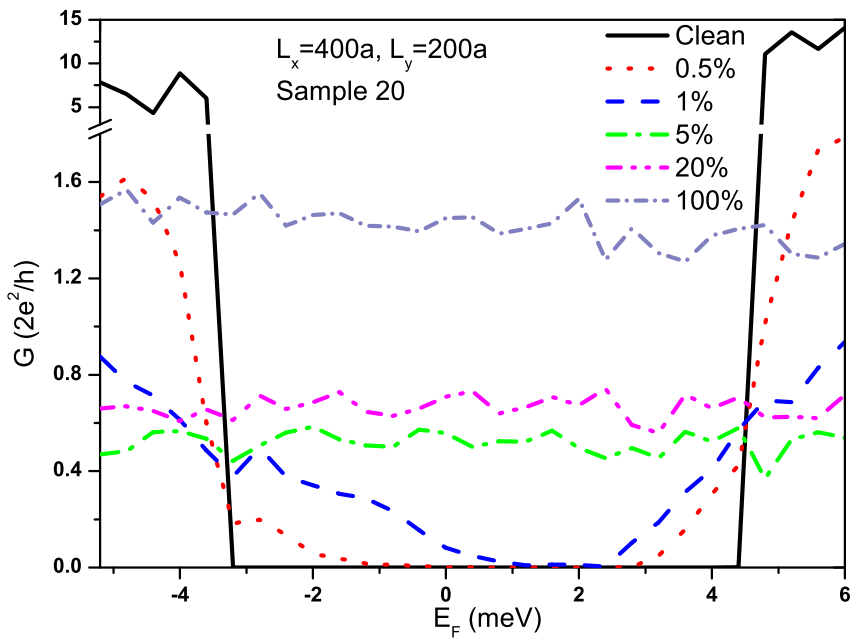

FIG. 6. (Color online) Bulk conductance changes with the impurity concentration. In the presence of dilute impurities, bulk conductance vanishes due to strong localization. As impurity concentration increases, wave function overlap between neighboring localized states will increase, resulting in finite bulk conductance.

As shown in Fig. 7, smooth disorder potential will give rise to in-gap states with localization length $\xi \gg L_{x}$, resulting in finite bulk conductance. These in-gap "extended" states

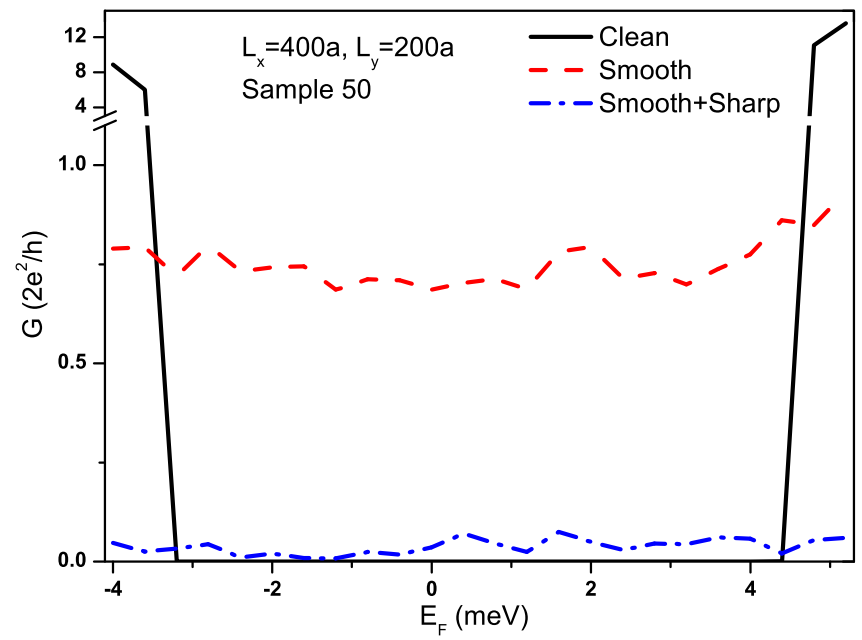

FIG. 7. (Color online) Bulk conductance in the presence of smooth disorder potential and smooth + sharp disorder potential. In-gap "extended" induced by smooth disorders will be localized by sharp disorders. The red dashed line is for the system with only smooth disorder. The blue dashed line is for the system with both smooth and sharp disorders.

will then be further localized by sharp impurity potential induced by dilute $\mathrm{Si}$ dopants, which significantly reduce the bulk conductance.
[1] C. L. Kane and E. J. Mele, Phys. Rev. Lett. 95, 226801 (2005).

[2] B. A. Bernevig and S. C. Zhang, Phys. Rev. Lett. 96, 106802 (2006).

[3] B. A. Bernevig, T. L. Hughes, and S. C. Zhang, Science 314, 1757 (2006).

[4] M. König, S. Wiedmann, C. Brune, A. Roth, H. Buhmann, L. W. Molenkamp, X. L. Qi, and S. C. Zhang, Science 318, 766 (2007).

[5] M. Z. Hasan and C. L. Kane, Rev. Mod. Phys. 82, 3045 (2010).

[6] X. L. Qi and S. C. Zhang, Rev. Mod. Phys. 83, 1057 (2011).

[7] J. E. Moore, Nature (London) 464, 194 (2010).

[8] A. Roth, C. Brüne, H. Buhmann, L. W. Molenkamp, J. Maciejko, X.-L. Qi, and S.-C. Zhang, Science 325, 294 (2009).

[9] C. X. Liu, T. L. Hughes, X.-L. Qi, K. Wang, and S.-C. Zhang, Phys. Rev. Lett. 100, 236601 (2008).

[10] I. Knez, R. R. Du, and G. Sullivan, Phys. Rev. Lett. 107, 136603 (2011).

[11] I. Knez, R. R. Du, and G. Sullivan, Phys. Rev. Lett. 109, 186603 (2012).

[12] K. Suzuki, Y. Harada, K. Onomitsu, and K. Muraki, Phys. Rev. B 87, 235311 (2013).

[13] Lingjie Du, Ivan Knez, Gerard Sullivan, and Rui-Rui Du, arXiv:1306.1925.
[14] M. Onoda, Y. Avishai, and N. Nagaosa, Phys. Rev. Lett. 98, 076802 (2007).

[15] Jian Li, Rui-Lin Chu, J. K. Jain, and Shun-Qing Shen, Phys. Rev. Lett. 102, 136806 (2009).

[16] Joseph Maciejko, Xiao-Liang Qi, and Shou-Cheng Zhang, Phys. Rev. B 82, 155310 (2010).

[17] Hua Jiang, Lei Wang, Qing-feng Sun, and X. C. Xie, Phys. Rev. B 80, 165316 (2009).

[18] C. W. Groth, M. Wimmer, A. R. Akhmerov, J. Tworzydlo, and C. W. J. Beenakker, Phys. Rev. Lett. 103, 196805 (2009).

[19] L. Fu and C. L. Kane, Phys. Rev. B 76, 045302 (2007).

[20] Peter Yu and Manuel Cardona, Fundamentals of Semiconductors: Physics and Materials Properties, 3rd ed. (Springer, Berlin, 2005).

[21] Cheung Chan and Tai-Kai Ng, Phys. Rev. B 85, 115207 (2012).

[22] A. MacKinnon, Z. Phys. B: Condens. Matter 59, 385 (1985); G. Metalidis and P. Bruno, Phys. Rev. B 72, 235304 (2005); G. Metalidis, Ph.D. thesis, Martin-Luther University at HalleWittenberg, Halle, 2007.

[23] R. Landauer, Philos. Mag. 21, 863 (1970); M. Büttiker, Phys. Rev. B 38, 9375 (1988); D. S. Fisher and P. A. Lee, ibid. 23, 6851 (1981).

[24] C. W. J. Beenakker, Rev. Mod. Phys. 69, 731 (1997).

[25] Bodo Huckestein, Rev. Mod. Phys. 67, 357 (1995). 